Research Article

\title{
Static and Dynamic Tensile Mechanical Behavior of Polyvinyl Chloride Elastomers with Different Shore Hardness
}

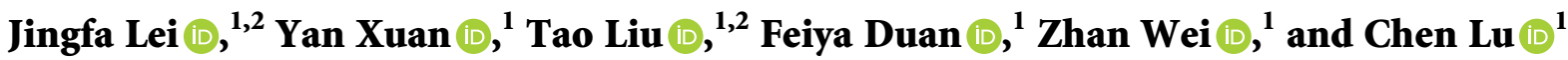 \\ ${ }^{1}$ School of Mechanical and Electrical Engineering, Anhui Jianzhu University, Hefei, China \\ ${ }^{2}$ Anhui Education Department Key Laboratory of Intelligent Manufacturing of Construction Machinery, Hefei, China
}

Correspondence should be addressed to Tao Liu; liutao19841015@163.com

Received 6 September 2020; Revised 17 January 2021; Accepted 20 February 2021; Published 9 March 2021

Academic Editor: Erkan Oterkus

Copyright (c) 2021 Jingfa Lei et al. This is an open access article distributed under the Creative Commons Attribution License, which permits unrestricted use, distribution, and reproduction in any medium, provided the original work is properly cited.

An experiment on the static and dynamic tensile mechanical properties of polyvinyl chloride (PVC) elastomers is conducted using an Instron-5943 universal testing machine and an improved Split Hopkinson Tensile Bar to study the dynamic tensile mechanical properties of PVC elastomer materials. The stress-strain curves of PVC materials with three types of Shore hardness (57A, 52A, and 47A) under the strain rates of $0.1 \mathrm{~s}^{-1}$ and $400 \sim 1800 \mathrm{~s}^{-1}$ are obtained. Results show that the mechanical behavior of PVC elastomer materials with different Shore hardness has remarkable linear elastic characteristics under the action of quasistatic tensile load. It has substantial sensitivity to strain rate and viscoelastic mechanical characteristics under the action of dynamic tensile load. The Zhu-Wang-Tang nonlinear viscoelastic constitutive model is used to characterize the viscoelastic mechanical characteristics with small error. This paper can provide theoretical model and method support for the design, development, production, and reliability analysis of PVC elastomers and other soft polymer materials.

\section{Introduction}

Polyvinyl chloride (PVC) is a plastic polymer with the second largest annual production worldwide. Its products/ components are widely used in vehicles [1], aviation [2], electronics [3], and other fields. This type of products/ components may bear dynamic impact loading during service. The mechanical properties, damage fracture, and failure characteristics of materials under dynamic impact loading are completely different from those under quasistatic loading. Compared with the compressive tests, tensile tests can provide more information on damage, fracture, and failure behavior. However, due to the experimental difficulties, the dynamic tensile behavior of PVC elastomers at high strain rates has been much less investigated. Therefore, the dynamic mechanical behavior of PVC elastomers should be studied.

The dynamic mechanical behavior of elastomer materials is more difficult to test than that of metal materials. The Split Hopkinson Pressure Bar (SHPB) is often used to study their dynamic compression mechanical behavior [4-8]. For example, A. Pellegrino et al. [9] have studied the mechanical response of syntactic foam with hollow glass microspheres in a polyurethane matrix via quasistatic and dynamic experiments. The material is considerably sensitive to the applied strain rate and exhibits high tensile and shear ductility at low and high strain rates. In the quasistatic experiment, the tension/compression asymmetry phenomenon occurs, but it disappears at high strain rate. Zhang et al. [10] have compared the mechanical properties of three types of STF capsules and obtained the best encapsulation method and formula. The introduction of UV curable resin can substantially improve the static strength of STF capsules. The STF capsule prepared through two-step polymerization has an elastic shell structure, which can withstand repeated impact without damage. For the dynamic tensile mechanical behavior test of soft polymer materials, there are still some problems, such as selection of specimen connection mode, low adhesive strength of adhesive, and unstable loading strain rate. Therefore, only a few scholars have studied the dynamic tensile mechanical response of soft polymer materials (clear flex 75 [7], PMMA [11], and silicone rubber 
[12]) by using the Split Hopkinson Tensile Bar (SHTB). Under dynamic loading, the response of polymers is elasticplastic with a hardening branch.

Researchers have studied the static and dynamic compression mechanical behavior of PVC elastomer materials. The research on the tensile mechanical behavior of PVC elastomers has focused mainly on the mechanical behavior under quasistatic loading, but the study on the dynamic tensile mechanical behavior of PVC elastomers is inadequate. The connection mode and adhesive of specimens are optimized via waveform analysis and using a high-speed camera in this study to reveal the static and dynamic tensile mechanical behavior of PVC elastomers with different Shore hardness. Constant strain rate loading is realized using a pulse shaper to delay the rising edge of incident wave, and the gap between incident and absorption rods is adjusted to solve the problem on the baseline deviation of incident wave. The static and dynamic tensile mechanical properties of PVC elastomer materials with three types of Shore hardness under the strain rates of $0.1 \mathrm{~s}^{-1}$ and $400 \sim 1800 \mathrm{~s}^{-1}$ are tested using an Instron-5943 universal testing machine and an improved SHTB device. The applicability of the material's nonlinear viscoelastic constitutive model has been verified.

\section{Experimental Materials and Methods}

2.1. Quasistatic Tensile Experiment. PVC elastomers with three types of Shore hardness (57A, 52A, and 47A) are used as experimental materials in this study [13]. The quasistatic tensile specimen is designed in accordance with SAC GB/T 528-200921 [14] and prepared by die stamping. Its specific dimension is shown in Figure 1; the dimension of the dynamic tensile specimen is $18 \times 2 \mathrm{~mm}$ [15].

The quasistatic tensile mechanical properties of the PVC elastomers are measured using an Instron-5943 universal testing machine at normal atmospheric temperature $\left(25^{\circ} \mathrm{C}\right)$. Both ends of the specimen are clamped in the upper and lower clamping chucks of the testing machine. An extensometer is added, as shown in Figure 2.

The quasistatic tensile test of a PVC elastomer with a strain rate of $0.1 \mathrm{~s}^{-1}$ is performed at normal atmospheric temperature $\left(25^{\circ} \mathrm{C}\right)$. The loading rate of the testing machine is $96 \mathrm{~mm} / \mathrm{min}$. The variations in time load $(F)$ and time shift $(\Delta L)$ in the tensile process are measured and recorded. Three groups of tensile loading experiments of the PVC elastomer are established, and the average values of load and shifting data are calculated. On the basis of (1), they are transformed into stress and strain data, and the stress-strain curves are obtained.

$$
\begin{aligned}
& \sigma=\frac{F}{A}, \\
& \mathcal{E}=\frac{\Delta L}{L_{0}},
\end{aligned}
$$

where $\sigma$ refers to stress, $A$ refers to the sectional area of the specimen, and $\varepsilon$ refers to strain.

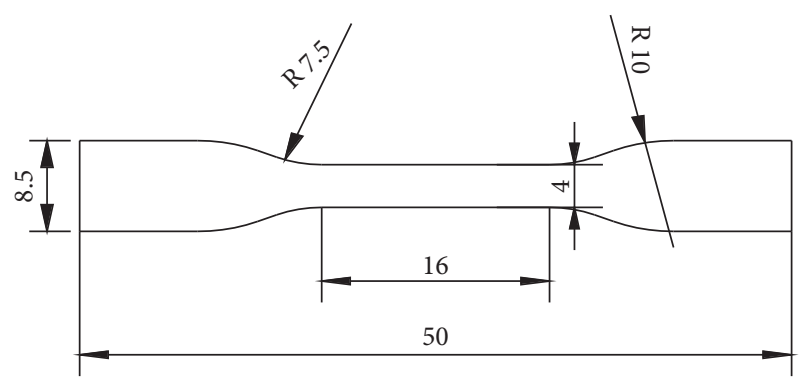

Figure 1: Dimension of the quasistatic tensile specimen.

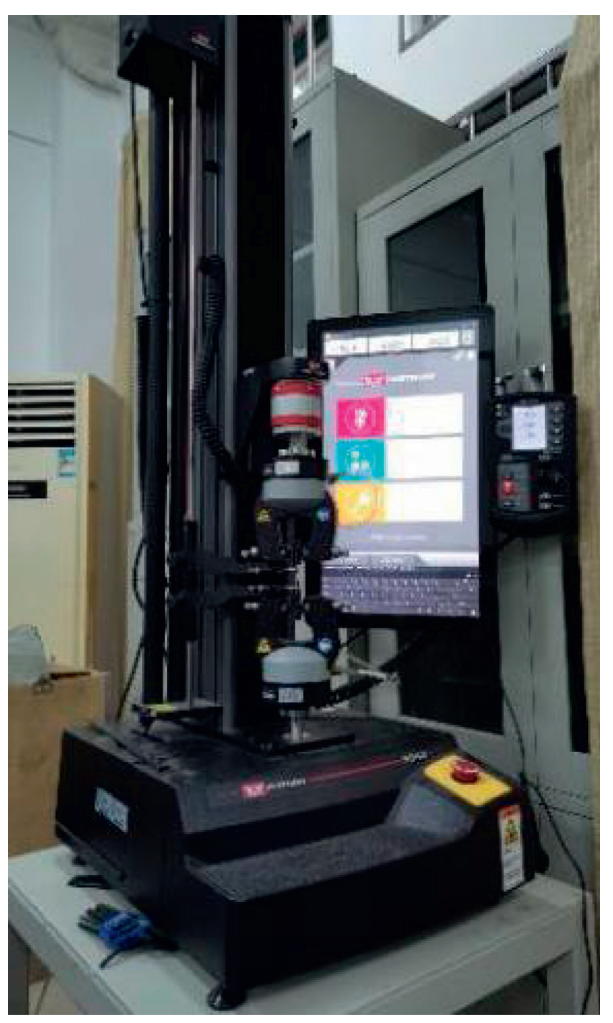

FIgURe 2: Quasistatic tensile experiment device.

\subsection{Dynamic Tensile Experiment}

2.2.1. SHTB Experimental Device and Principle. In this study, a solid aluminum SHTB with a diameter of $20 \mathrm{~mm}$ is used to conduct the dynamic tensile experiment of PVC elastomer materials at high strain rate. The total length of the bar system is $11500 \mathrm{~mm}$. Among them, the incident bar is $5000 \mathrm{~mm}$ long, the transmission bar is $5000 \mathrm{~mm}$ long, and the absorption bar is $1500 \mathrm{~mm}$ long. A flange is mounted on the incident bar and can move only a small distance due to the restriction of insulating tape. The tubular aluminum sleeve bullet is $600 \mathrm{~mm}$ long. The principle and material object of the SHTB device are shown in Figure 3.

In the SHTB experimental device, semiconductor strain gauges are used in the incident and transmission bars to improve the sensitivity of the pulse signal and obtain incident, reflected, and transmitted pulses. Gas is charged into the gas storage chamber by rotating the valve of the gas 


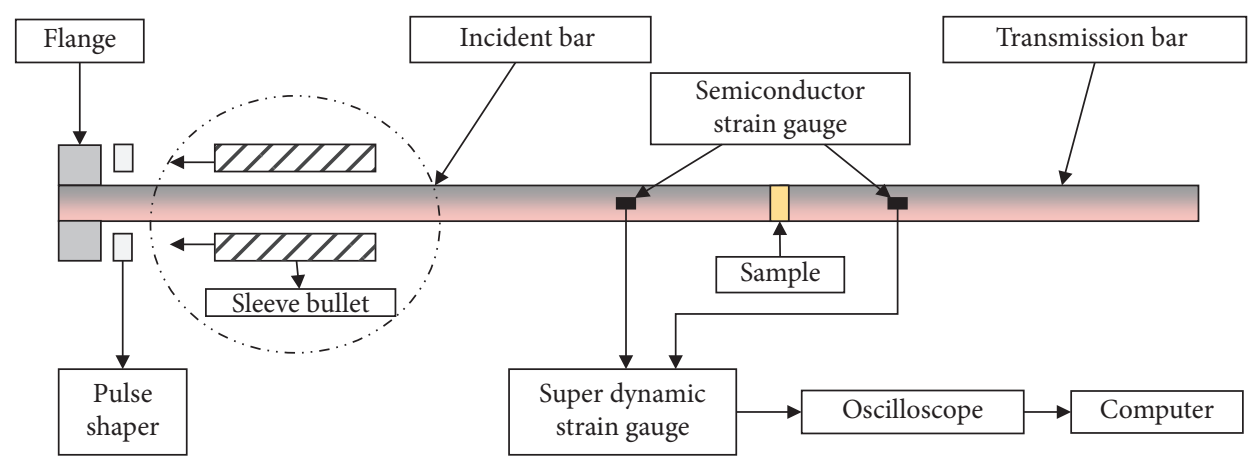

(a)

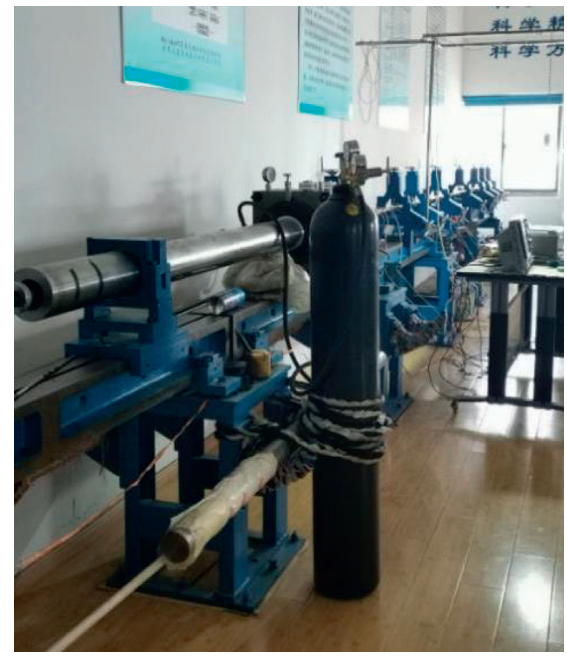

(b)

FIgURE 3: Principle and material object of the SHTB device.

cylinder. When the air pressure in the gas storage chamber is about to reach the predetermined value, the valve of the gas storage chamber is rapidly tightened. The launching valve on the launching chamber is pressed to the lowest position and kept at that position for a period of time to make the highpressure gas in the launching chamber flow out rapidly and stably. The sleeve bullet is pushed to accelerate its movement on the incident bar for a certain distance and then impact the flange plate at its left end. After the impact, the right-hand tensile wave is generated on the incident bar $\varepsilon_{i}(\tau)$. When the tensile wave $\varepsilon_{i}(\tau)$ propagates to the place where the right end of the incident bar connects to the tensile specimen, it is divided into two parts. One part refers to the reflected waves $\varepsilon_{r}(\tau)$ propagating to the left in the incident bar; the other part refers to $\varepsilon_{t}(\tau)$ propagating to the right in the transmission bar. Among them, the pulse signals of incident wave $\varepsilon_{i}(\tau)$ and reflected wave $\varepsilon_{r}(\tau)$ are recorded using the strain gauge on the incident bar. The pulse signal of transmission wave $\varepsilon_{t}(\tau)$ is recorded using the strain gauge on the transmission bar. Without considering the generation mode of incident pulse, the experimental principle of SHTB is completely the same as that of SHPB, and both satisfy the dynamic mechanical properties under the one-dimensional stress wave hypothesis and uniformity hypothesis [16]. In accordance with one-dimensional stress wave theory, the relationships among average stress, strain, and strain rate are obtained and transformed into stress-strain curves.

$$
\begin{aligned}
& \sigma_{e}=\frac{A E \varepsilon_{T}}{A_{S}} \\
& \varepsilon_{e}=\int_{0}^{t} \frac{2 C_{0} \varepsilon_{R}}{L_{s}} \mathrm{~d} t, \\
& \dot{\varepsilon}_{e}=\frac{2 C_{0} \varepsilon_{R}}{L_{S}},
\end{aligned}
$$

where $\sigma_{e}, \varepsilon_{e}$, and $\dot{\varepsilon}_{\mathrm{e}}$ refer to the average stress, strain, and strain rate, respectively; $A$ is the cross-sectional area of the sample; $C_{0}$ refers to the one-dimensional elastic wave velocity of the bar; and $L_{S}$ refers to the length of the cylindrical specimen.

2.2.2. Key Technique of SHTB Experiment. Three connection modes of specimens, namely, thread, clamp, and adhesive connections, occur in the SHTB experiment. A PVC elastomer is difficult to be processed into a cylindrical dumbbellshaped specimen that can meet the experimental requirements because of its low strength. The existence of thread gap and the clamping structure itself will affect the 
propagation of stress wave. Therefore, the dynamic tensile test specimens shall not be connected using thread or clamp but by adhesive connection. The dimension of specimens with adhesive connection is $18 \times 2 \mathrm{~mm}$. Complete consolidation can make the adhesive reach the maximum strength. Therefore, 502 instant super glue with high strength and short consolidation time (approximately $10 \mathrm{~min}$ ) is used in this study to accelerate the experiment. The loading waveform obtained in this experiment is shown in Figure 4.

Figure 4 depicts that the incident pulse remains a rectangular square wave and can reach the maximum strain within $100 \mu \mathrm{s}$. The transmission pulse rises to the maximum strain in a short time and then decreases rapidly to the baseline. The dynamic tensile experiment process recorded using a v1212 high-speed camera is observed (Figure 5) to determine the reason why the transmitted wave is unloaded immediately after reaching the maximum strain in Figure 4. When the wave is transmitted to the bonding surface between the specimen and incident bar, the 502 super glue shakes violently, resulting in the debonding of the bonding surface between the specimen and incident bar. Consequently, the transmitted wave is unloaded immediately after it reaches the maximum strain. The specific tensile comparison is shown in Figure 5(b).

Many types of adhesives, such as YH-818 rubber glue for special purpose, Guteling 401 glue, epoxy resin AB glue, and JL-330 special rubber adhesive, are studied to solve the abovementioned problems. Only the epoxy resin $A B$ glue and JL-330 special rubber adhesive can firmly bond the specimen between the incident and transmission bars. A dynamic tensile experiment is conducted on the specimens bonded using the two adhesives by using the SHTB device to confirm the applicability of the two adhesives. The experimental waveform obtained in the experiment is shown in Figure 6.

Figure 6 illustrates that the obtained incident pulses still show a rectangular square wave and can reach the maximum strain within $100 \mu \mathrm{s}$. Among them, the transmission pulse of the bonding specimen by using epoxy resin $\mathrm{AB}$ glue rises to the maximum strain and then decreases rapidly to the baseline. The loading time of the transmission pulse of the boding specimen by using JL-330 special rubber adhesive becomes longer (approximately $200 \mu \mathrm{s}$ ), reaches the maximum, and slowly decreases. This result indicates that the JL330 special rubber glue can alleviate the problem on unloading immediately after the transmission wave rises to the maximum strain. Therefore, we can perform a dynamic tensile test of PVC elastomers by using JL-330 special rubber glue in the subsequent research.

When direct loading is adopted in the SHTB experiment, the incident wave is a square wave and can reach the maximum strain in a short time, which cannot guarantee constant strain rate loading in the entire loading process. To ensure the accuracy of the SHTB experimental results, a pulse shaper is placed on the end face of the flange (the end face impacted by the sleeve bullet) to obtain the characteristics of the incident wave required (the loading time corresponding to the width of the rising edge of the incident wave is ensured to be greater than the time required for three

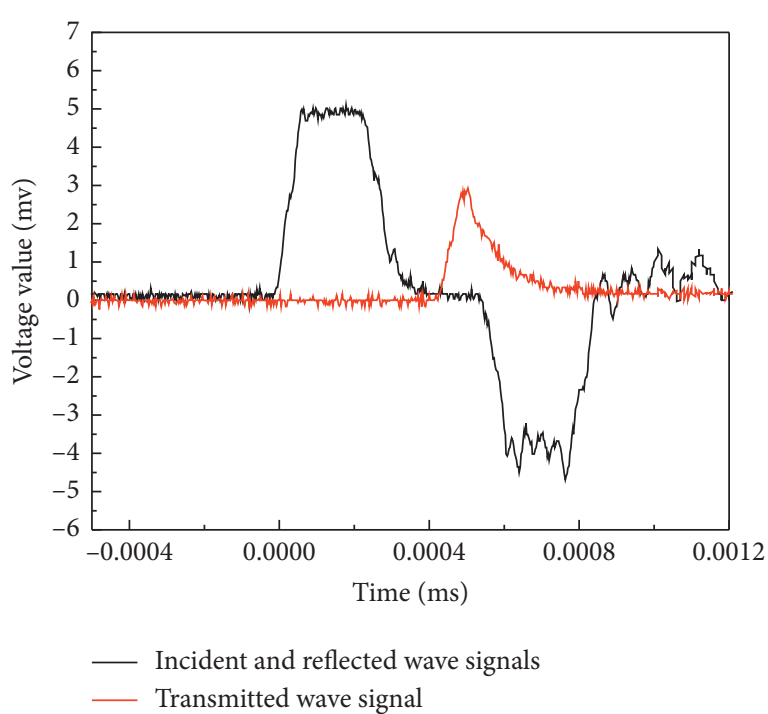

FIGURE 4: Loading waveform of the improved SHTB adhesive specimen.

times of intercourse of the loading pulse in the specimen [13]) and realize constant strain rate loading. The raw material of the pulse shaper is postcard paper. It is processed into a ring-shaped piece with (outer diameter) $28.4 \mathrm{~mm} \times$ (inner diameter) $20 \mathrm{~mm}$ by using a special mold. It is adhered to the end face of the flange by applying a small amount of Vaseline. The waveform after shaping is shown in Figure 7.

Figure 7 demonstrates that the rising edge of the incident wave after shaping is approximately $270 \sim 300 \mu$ s, and three times of intercourse of stress wave propagating in the specimen (the wave velocity of the specimen is approximately $50 \mathrm{~m} / \mathrm{s}$ ) takes only $240 \mu \mathrm{s}$. Therefore, the stress equilibrium state can be achieved in the specimen. On the basis of the previous experience on the split Hopkinson bar experiment, a certain gap $(2 \sim 5 \mathrm{~mm})$ is often left between absorption and incident bars to avoid the secondary impact between the bars. Nevertheless, in Figure 8, the incident wave is incompletely unloaded to the baseline. The analysis of the experimental device shows that the main reason why the incident wave deviates from the baseline is that the flange thickness is considerably less than the bullet length, which makes the bullet and flange move together. To solve this problem, the gap between incident and absorption bars should be eliminated to unload the incident wave and obtain the ideal experimental waveform, as shown in Figure 8.

\section{Experimental Results and Analysis}

3.1. Analysis on Quasistatic Tensile Mechanical Properties. The tensile stress-strain curves of PVC elastomers with three types of Shore hardness (57A, 52A, and 47A) at a low strain rate $\left(0.1 \mathrm{~s}^{-1}\right)$ are shown in Figure 9 .

Figure 9 indicates that the stress of PVC elastomers with three types of Shore hardness at a low strain rate $\left(0.1 \mathrm{~s}^{-1}\right)$ under uniaxial tensile loading increases with the increase in strain, showing a linear change in general. The tensile elastic 


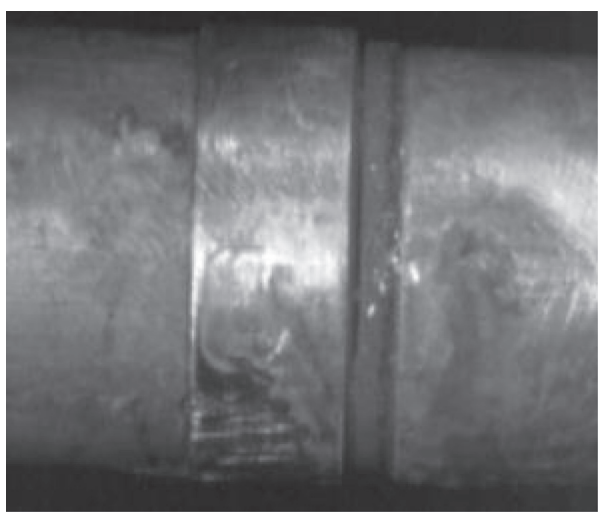

(a)

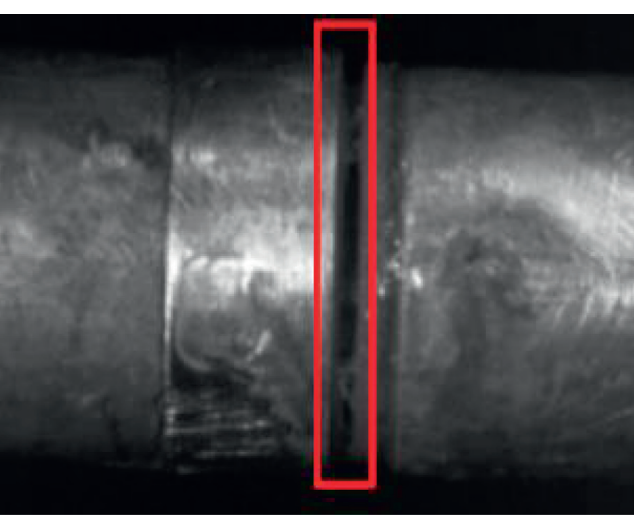

(b)

FIGURE 5: Comparison of the stretching of the bonding specimen by using 502 super glue. (a) Before stretching. (b) The moment when wave transmits to the specimen.

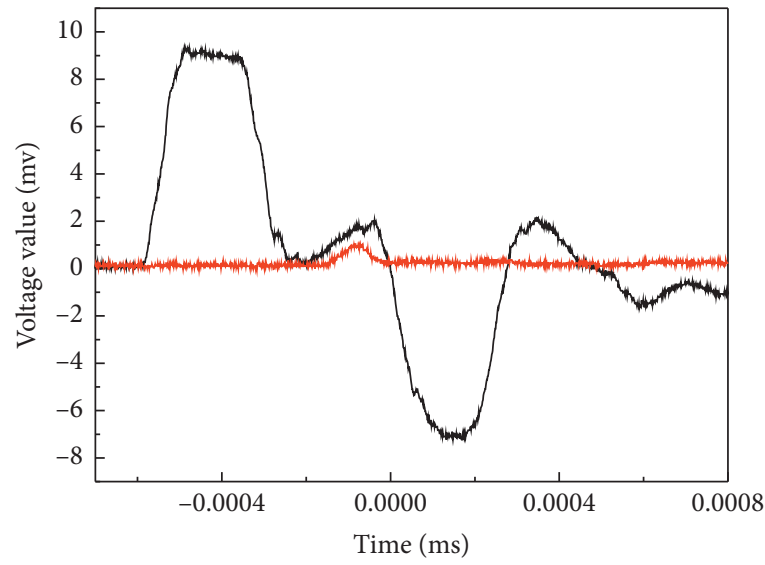

_ Incident and reflected wave signals

— Transmitted wave signal

(a)

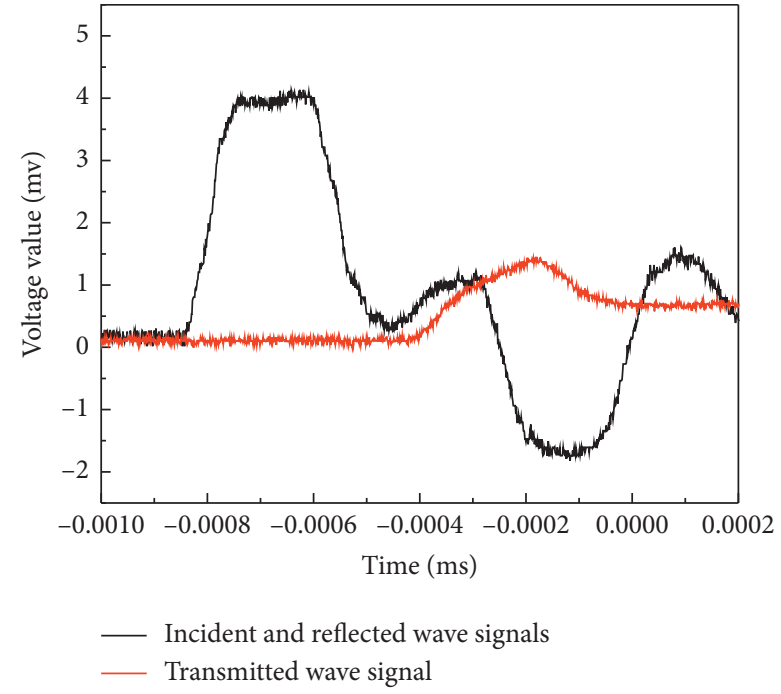

(b)

FIGURE 6: Typical waveform of bonding by using different adhesives. (a) Waveform of the epoxy resin AB glue. (b) Waveform of the JL-330 special rubber adhesive.

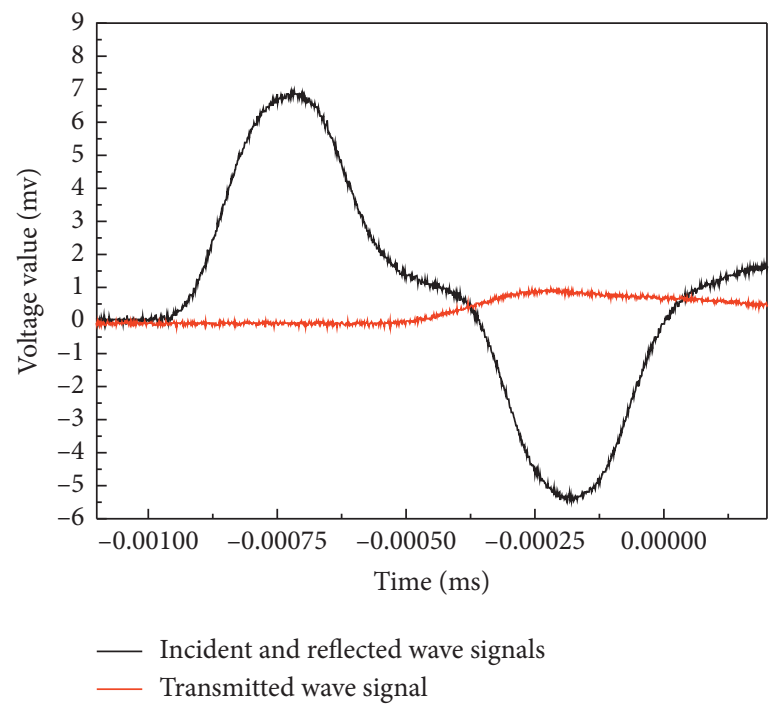

FIgURE 7: Loading waveform after shaping. 


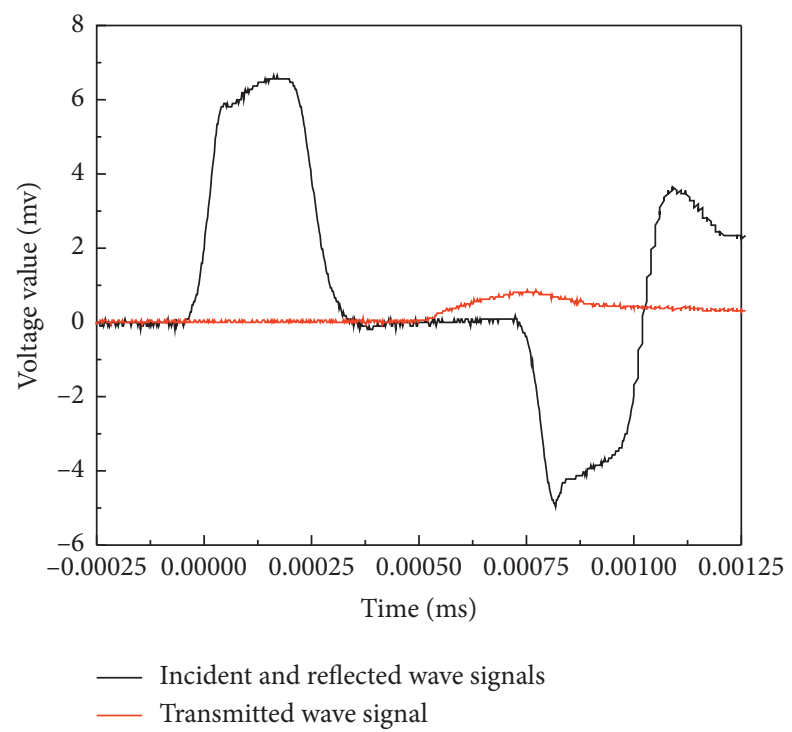

FIGURE 8: Waveform with no gap between incident and absorption bars.

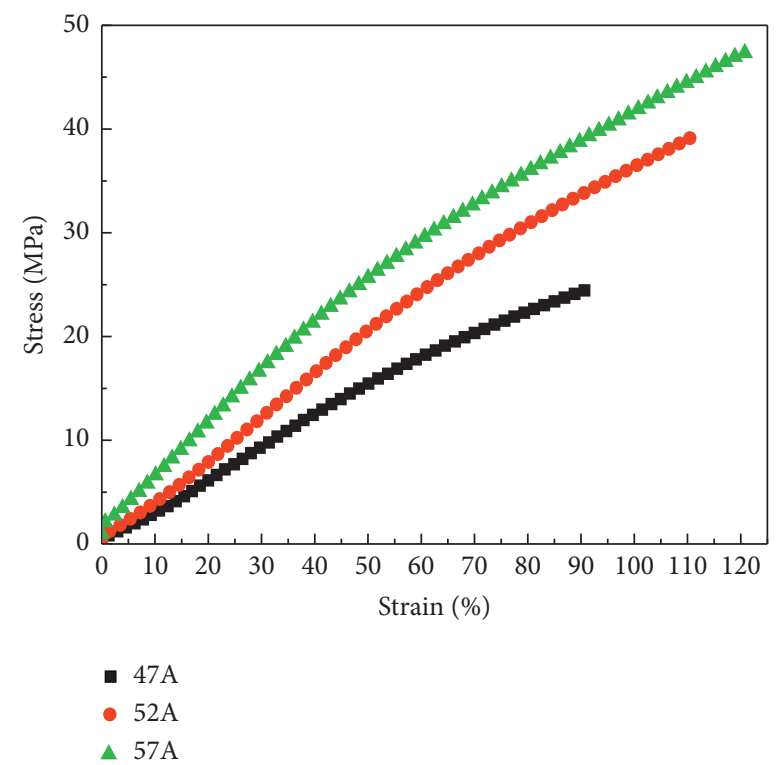

FIGURE 9: Quasistatic tensile stress-strain curves of PVC elastomers with three types of Shore hardness.

modulus of the three PVC elastomers at the strain rate of $0.1 \mathrm{~s}^{-1}(43.2,33.4$, and $27.0 \mathrm{MPa})$ increases with the increase in Shore hardness.

3.2. Analysis of Dynamic Tensile Mechanical Properties. The stress-strain curves of PVC elastomers with three types of Shore hardness at the strain rate of $400 \sim 1800 \mathrm{~s}^{-1}$ can be obtained through processing the dynamic tensile experimental data, as shown in Figures 10(a)-10(c).

Figure 10 shows that the stress-strain curves of PVC elastomers with three types of Shore hardness under highstrain-rate tensile loading are divided into three stages, namely, elastic, plastic, and unloading, implying evident nonlinear characteristics. In addition, they have different tensile elastic modulus, peak stress, and peak strain under varying high-strain-rate tensile loading, showing certain strain rate sensitivity.

In Figure 10, the corresponding point at the strain rate of $0.1 \mathrm{~s}^{-1}$ is connected with the original point. Young's modulus $E_{g}$ is characterized by its slope, which is called secant modulus. The secant modulus, peak stress, and peak strain corresponding to the strain rate of $0.1 \mathrm{~s}^{-1}$ in Figure 10 are compared to explain the strain rate sensitivity of PVC elastomers with three types of Shore hardness. The specific changes are shown in Table 1.

Table 1 presents that the secant modulus, peak stress, and peak strain of the PVC elastomers with three types of Shore 


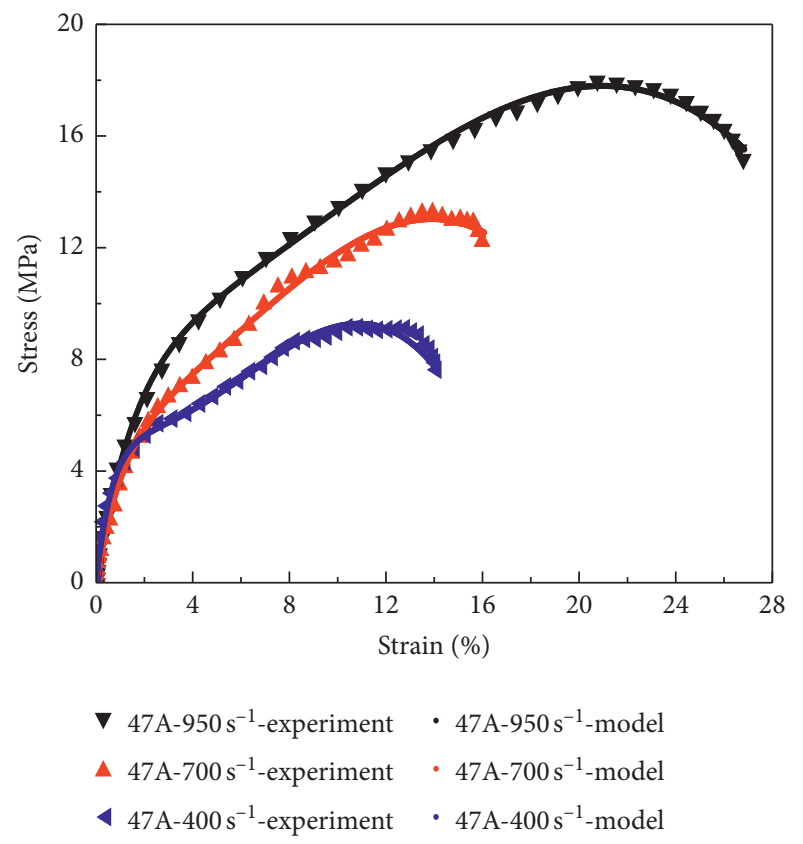

(a)

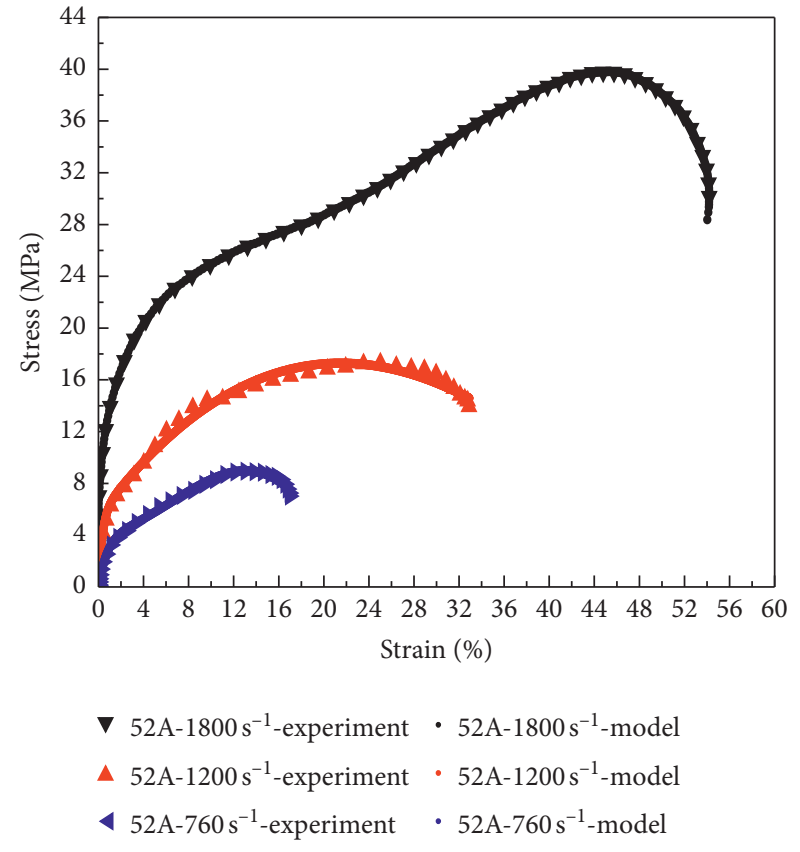

(b)

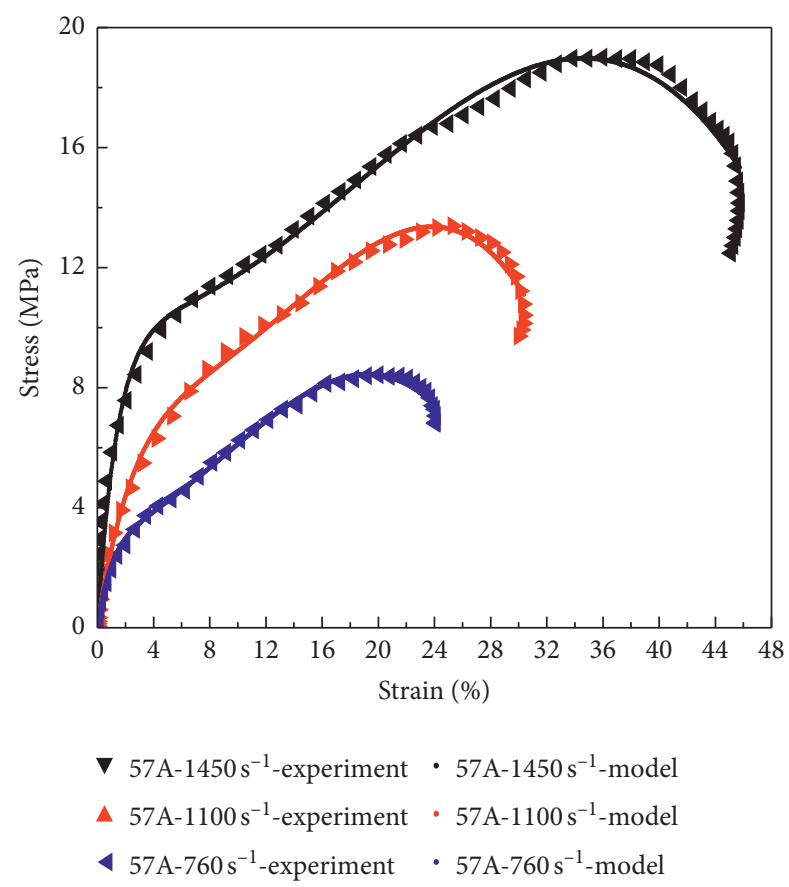

(c)

FIgURe 10: Dynamic tensile stress-strain curves of PVC elastomers with three types of Shore hardness.

TABle 1: Table 1Changes in dynamic tensile parameters.

\begin{tabular}{|c|c|c|c|c|c|c|c|c|c|}
\hline & \multicolumn{3}{|c|}{$57 \mathrm{~A}$} & \multicolumn{3}{|c|}{$52 \mathrm{~A}$} & \multicolumn{3}{|c|}{$47 \mathrm{~A}$} \\
\hline & $760 s^{-1}$ & $1100 \mathrm{~s}^{-1}$ & $1450 \mathrm{~s}^{-1}$ & $760 \mathrm{~s}^{-1}$ & $1200 \mathrm{~s}^{-1}$ & $1800 \mathrm{~s}^{-1}$ & $400 s^{-1}$ & $700 s^{-1}$ & $950 \mathrm{~s}^{-1}$ \\
\hline Secant modulus (MPa) & 61.88 & 95.48 & 119.61 & 81.90 & 90.85 & 250.51 & 89.78 & 115.62 & 134.82 \\
\hline Peak stress $(\mathrm{MPa})$ & 8.43 & 13.38 & 19.02 & 8.97 & 18.12 & 40.40 & 9.19 & 13.25 & 17.99 \\
\hline Peak strain (\%) & 24.10 & 25.37 & 45.74 & 17.00 & 32.91 & 54.20 & 14.12 & 16.07 & 26.85 \\
\hline
\end{tabular}




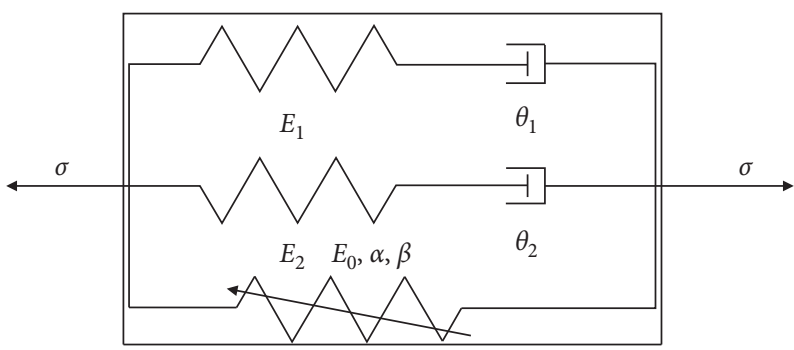

FIGURE 11: ZWT model sketch.

TABle 2: Parameters of the dynamic tensile strain curve fitted using the ZWT model.

\begin{tabular}{lccccccccc}
\hline & \multicolumn{3}{c}{$47 \mathrm{~A}$} & & $52 \mathrm{~A}$ & & \multicolumn{2}{c}{$57 \mathrm{~A}$} \\
& $400 \mathrm{~s}^{-1}$ & $700 \mathrm{~s}^{-1}$ & $950 \mathrm{~s}^{-1}$ & $760 \mathrm{~s}^{-1}$ & $1200 \mathrm{~s}^{-1}$ & $1800 \mathrm{~s}^{-1}$ & $760 \mathrm{~s}^{-1}$ & $1100 \mathrm{~s}^{-1}$ & $1450 \mathrm{~s}^{-1}$ \\
\hline$E_{0}(\mathrm{MPa})$ & -7.3644 & 8557 & 51.2489 & 2.7257 & 183.0922 & -1.3953 & 6.8999 & 1.5411 & -3.8991 \\
$E_{1}(\mathrm{MPa})$ & 6.9296 & -8556 & -51.1514 & -2.7488 & -181.9319 & 1.2037 & -6.8335 & -2.2823 & 3.7993 \\
$\alpha(\mathrm{MPa})$ & 0.1663 & 0.0761 & 0.0507 & 0.0852 & -0.0315 & 0.0385 & 0.03559 & 0.0730 & 0.0268 \\
$\beta(\mathrm{MPa})$ & -0.0090 & -0.0041 & -0.0017 & -0.0043 & 0.0001 & -0.0006 & -0.0013 & -0.0016 & -0.0005 \\
$E_{2}(\mathrm{MPa})$ & 8.4140 & 5.0744 & 5.4817 & 5.1286 & 15.5890 & 0.0035 & 2.9987 & 3.8203 & 7.3353 \\
$\theta_{2}(\mathrm{MPa})$ & 0.0017 & 0.0016 & 0.0017 & 0.0011 & 0.0003 & 0.0005 & 0.0014 & 0.0027 & 0.0010 \\
\hline
\end{tabular}

hardness under tensile loading with strain rates of $760 \sim 1450 \mathrm{~s}^{-1}, \quad 760 \sim 1800 \mathrm{~s}^{-1}$, and $400 \sim 950 \mathrm{~s}^{-1}$ increase with the increase in strain rate.

\section{Zhu-Wang-Tang (ZWT) Nonlinear Viscoelastic Model}

The ZWT nonlinear viscoelastic model [17] is a widely used model to describe the nonlinear viscoelastic behavior of polymer materials in the strain rate range of $10^{-4} \sim 10^{3} \mathrm{~s}^{-1}$ proposed by Zhu Zhaoxiang, Wang Lili, and Tang Zhiping after many years of research on engineering plastics (such as polycarbonate, nylon, and epoxy resin). This model is composed of one nonlinear spring and two Maxwell units in parallel (Figure 11). Its constitutive expression is as follows.

$$
\begin{aligned}
\sigma= & E_{0} \varepsilon+\alpha \varepsilon^{2}+\beta \varepsilon^{3}+E_{1} \int_{0}^{t} \dot{\varepsilon} \exp \left(-\frac{t-\tau}{\theta_{1}}\right) \mathrm{d} \tau \\
& +E_{2} \int_{0}^{t} \dot{\varepsilon} \exp \left(-\frac{t-\tau}{\theta_{2}}\right) \mathrm{d} \tau
\end{aligned}
$$

In (4), the first three items are used to characterize the nonlinear elastic response of the material, which is independent of the strain rate. $E_{0}, \alpha$, and $\beta$ refer to the elastic constants of nonlinear spring. The first Maxwell unit (Item 4) can be used to describe the viscoelastic behavior of polymer materials under low-strain-rate loading. $E_{1}$ refers to its elastic constant, and $\theta_{1}$ refers to its relaxation time. The second Maxwell unit (Item 5) can be used to describe the viscoelastic behavior of polymer materials under highstrain-rate loading. $E_{2}$ refers to its elastic constant, and $\theta_{2}$ refers to its relaxation time.

When a material is subjected to a low-strain-rate loading, the Maxwell unit, which describes its viscoelastic behavior at high strain rate, is always in a relaxed state during loading. Therefore, the expression of the ZWT nonlinear viscoelastic model to describe the viscoelastic behavior of materials at low strain rate is as follows:

$$
\sigma=E_{0} \varepsilon+\alpha \varepsilon^{2}+\beta \varepsilon^{3}+E_{1} \int_{0}^{t} \dot{\varepsilon} \exp \left(-\frac{t-\tau}{\theta_{1}}\right) \mathrm{d} \tau .
$$

Given that low strain rates are mostly constant strain rates, (5) can be rewritten as

$$
\sigma=E_{0} \varepsilon+\alpha \varepsilon^{2}+\beta \varepsilon^{3}+E_{1} \theta_{1} \dot{\varepsilon}\left(1-\exp \left(-\frac{\varepsilon}{\theta_{1} \dot{\varepsilon}}\right)\right) .
$$

When a material is under the action of high-strain-rate loading, the dashpot in the Maxwell element, which describes its viscoelastic behavior at low strain rate, has no time to relax due to the excessively short loading time. As a result, the Maxwell element can only play the role of spring. The expression of the ZWT nonlinear viscoelastic model to describe the viscoelastic behavior of materials at high strain rate is as follows:

$$
\sigma=\left(E_{0}+E_{1}\right) \varepsilon+\alpha \varepsilon^{2}+\beta \varepsilon^{3}+E_{2} \int_{0}^{t} \dot{\varepsilon} \exp \left(-\frac{t-\tau}{\theta_{2}}\right) \mathrm{d} \tau
$$

In the dynamic tensile experiment of PVC elastomers with three types of Shore hardness, the loading strain rate can be approximately regarded as a constant strain rate. Therefore, (8) can be rewritten as

$$
\sigma=\left(E_{0}+E_{1}\right) \varepsilon+\alpha \varepsilon^{2}+\beta \varepsilon^{3}+E_{2} \theta_{2} \dot{\varepsilon}\left(1-\exp \left(-\frac{\varepsilon}{\theta_{2} \dot{\varepsilon}}\right)\right) \text {. }
$$

Equation (8) is used to fit the experimental data of the PVC elastomers with three types of Shore hardness under high-strain-rate tensile loading, and the parameters obtained 
are shown in Table 2. The corresponding fitting curve is compared with the test curve, as shown in Figure 10.

Figure 10 shows a comparison of the dynamic tensile experimental result and the ZWT model fitting result. Both are in good agreement. This finding indicates that the constitutive model can well describe the viscoelastic mechanical properties of the PVC elastomers with three types of Shore hardness under high-strain-rate tensile loading.

\section{Conclusion}

In this study, a tensile experiment is performed for PVC elastomers with three types of Shore hardness ( $57 \mathrm{~A}, 52 \mathrm{~A}$, and $47 \mathrm{~A})$ under quasistatic $\left(0.1 \mathrm{~s}^{-1}\right)$ and dynamic (400 1800 $\mathrm{s}^{-1}$ ) loading by using an Instron-5943 universal testing machine and an improved SHTB experimental device. The stress-strain curve is obtained. Its strain rate sensitivity is also analyzed.

(1) The dynamic tensile technique based on the SHTB device proposed in this paper, which can meet two basic hypotheses of Hopkinson experiment, can be used in soft polymer materials. The experimental result can reflect the dynamic mechanical properties of the materials, and the device structure is simple and easy to operate and popularize.

(2) The tensile mechanical behavior of PVC elastomers has obvious linear elastic characteristics at low strain rate $\left(0.1 \mathrm{~s}^{-1}\right)$ under tensile loading and evident strain rate sensitivity at high-strain-rate $\left(400 \sim 1800 \mathrm{~s}^{-1}\right)$ loading.

(3) PVC elastomers present obvious viscoelastic characteristics of dynamic tensile mechanical properties and the characteristic error described by the ZWT constitutive model.

The conclusions obtained in this study can provide a reference for the research on the static and dynamic mechanical properties of soft polymer materials. In the later stage, we will study the influence of ambient temperature on the static and dynamic mechanical responses of soft polymer materials and construct a viscoelastic-hyperelastic constitutive model with strain rate as the only variable. Such a study will provide theoretical model and method support for the design and development of PVC elastomers and other soft polymer materials.

\section{Data Availability}

The data used to support the findings of this study are available from the corresponding author upon request.

\section{Conflicts of Interest}

The authors declare no conflicts of interest.

\section{Acknowledgments}

This work was supported by Anhui Education Department Top-Notch Talents Academic Funding Project (Grant no.
GxbjZD2020078), Anhui Education Department Excellent Young Talent Support Project (Grant no. GxyqZD2019057), Anhui Province Key Research and Development Project (Grant no. 1804a09020009), and Natural Science Foundation of Anhui Province (Grant no. 1708085ME130).

\section{References}

[1] H. Chao, E. Su, Q. Yan, and W. Huang, "An experimental study on dynamic characteristics of two rubber-type engine vibration isolators," Experimental Mechanics, vol. 34, no. 1, pp. 157-165, 2019.

[2] W. Wang, W. Li, P. Liang, Y. Xu, and T. Xu, "Preparation of vehicle crash dummy skin and its mechanical property testing," Journal of Jilin University (Engineering Edition), vol. 49, no. 1, pp. 192-198, 2019.

[3] B. Du, X. Kong, X. Meng, Li Jin, and Z. Qian, "Research progress of high thermal conductivity polymer matrix composites," Transactions of China Electrotechnical Society, vol. 33, no. 14, pp. 3149-3159, 2018.

[4] G. Chen, Q. Zhang, and X. Huang, "SHPB waveform shaping technology based on soft materials," Chinese Science: Technical Science, vol. 46, no. 4, pp. 393-399, 2016.

[5] $\mathrm{H}$. Wu and X. Jiang, "Dynamic mechanical properties of polyurethane foam under low strain rate and large strain," Explosion and Shock Waves, vol. 31, no. 4, pp. 392-396, 2011.

[6] B. Xie, L. Tang, X. Jiang et al., "SHPB system driven by electromagnetic force of double bullets for soft materials," Explosion and Shock Waves, vol. 39, no. 5, pp. 69-75, 2019.

[7] J. T. Fan, J. Weerheijm, L. J. Sluys et al., "Deformation to fracture evolution of a flexible polymer under split Hopkinson pressure bar loading," Polymer Testing, vol. 70, pp. 192-196, 2018.

[8] A. Ahmed, N. Asija, H. Chauhan et al., "Study of polycarbonate based nano-composites at high strain rate impact," Procedia Structural Integrity, vol. 14, pp. 507-513, 2019.

[9] A. Pellegrino, V. L. Tagarielli, and R. Gerlach, "The mechanical response of a syntactic polyurethane foam at low and high rates of strain," International Journal of Impact Engineering, vol. 75, pp. 214-221, 2015.

[10] X. Zhang, H. Zhang, and P. Wang, "Optimization of shear thickening fluid encapsulation technique and dynamic response of encapsulated capsules and polymeric composite," Composites Science and Technology, vol. 170, pp. 165-173, 2019.

[11] H. Wu, G. Ma, and Y. Xia, "Experimental study of tensile properties of PMMA at intermediate strain rate," Materials Letters, vol. 58, pp. 3681-3685, 2004.

[12] L. Guo, Y. Lv, and Z. Deng, "Tension testing of silicone rubber at high strain rates," Polymer Testing, vol. 50, pp. 270-275, 2016.

[13] J. Lei, X. Yan, and T. Liu, "Static and dynamic mechanical behavior and constitutive model of PVC elastomers for design processes of soft polymer materials," Advances in Mechanical Engineering, vol. 12, no. 6, pp. 1-12, 2020.

[14] Standardization Administration of China, GB/T 528-2009 Rubber, Vulcanized or Thermoplastic-Determination of Tensile Stress-Strain Properties, Standards Press of China, Beijing, China, 2009.

[15] Y. Zhou, Li Chen, and S. Cui, "A new technique of dynamic direct tensile experiment for soft materials," Journal of $\mathrm{Vi}$ bration and Shock, vol. 36, no. 22, pp. 144-148+224, 2017.

[16] M. Saleh, M. M. Kariem, V. Luzina et al., "High strain rate deformation of ARMOX 500T and effects on texture 
development using neutron diffraction techniques and SHPB testing," Materials Science and Engineering: A, vol. 709, pp. 30-39, 2018.

[17] H. Zhou, S. Li, K. Xie, S. Xin, and L. Yang, "Research on the viscoelastic constitutive model of HTPB propellant over a wide range of strain rates," Journal of Solid Rocket Technology, vol. 40, no. 3, pp. 325-329+39, 2017. 